\title{
How granular vortices can help understanding rheological and mixing prop- erties of dense granular flows
}

\author{
Pierre Rognon ${ }^{1, \star}$, Prashidha Kharel ${ }^{1, \star \star}$, Thomas Miller ${ }^{1, \star \star \star}$, and Itai Einav ${ }^{1,2, \star \star \star \star}$ \\ ${ }^{1}$ Particles and Grains Laboratory, School of Civil Engineering, The University of Sydney, Sydney, New South Wales 2006, Australia. \\ ${ }^{2}$ Department of Civil, Environmental \& Geomatic Engineering, University College London, London WC1E 6BT, UK.
}

\begin{abstract}
Dense granular flows exhibit fascinating kinematic patterns characterised by strong fluctuations in grain velocities. In this paper, we analyse these fluctuations and discuss their possible role on macroscopic properties such as effective viscosity, non-locality and shear-induced diffusion. The analysis is based on 2D experimental granular flows performed with the stadium shear device and DEM simulations. We first show that, when subjected to shear, grains self-organised into clusters rotating like rigid bodies. The average size of these so-called granular vortices is found to increase and diverge for lower inertial numbers, when flows decelerate and stop. We then discuss how such a microstructural entity and its associated internal length scale, possibly much larger than a grain, may be used to explain two important properties of dense granular flows: (i) the existence of shear-induced diffusion of grains characterised by a shear-rate independent diffusivity and (ii) the development of boundary layers near walls, where the viscosity is seemingly lower than the viscosity far from walls.
\end{abstract}

\section{Introduction}

Dense granular flows involved in geophysical processes and industrial applications are typically comprised of a considerable number of grains. Often, a continuum description of their rheological behaviour and mixing properties is required, as predicting the dynamics of every individual grains would exceed the best computational capabilities. However, continuum-based predictions critically rely on effective parameters such as effective viscosity and effective diffusivity. It is therefore necessary to establish reliable and accurate models rationalising how these parameters depend on the grain properties and on the flow conditions.

Effective viscosity $v\left[\mathrm{~m}^{2} / \mathrm{s}\right]$ and diffusivity $D\left[\mathrm{~m}^{2} / \mathrm{s}\right]$ both involve a length scale and a time scale. Considering grains of size $d[\mathrm{~m}]$ subjected to a shear strain rate $\dot{\gamma}\left[\mathrm{s}^{-1}\right]$ naturally leads to expressing the following models, referred to as Bagnold's scaling [1-4]:

$$
\begin{aligned}
D & \propto d^{2} \dot{\gamma} \\
v & \propto d^{2} \dot{\gamma}
\end{aligned}
$$

While these scalings are generally valid, there are multiple evidences pointing out that they are not always sufficient to capture the observed rheological and mixing behaviour. Specifically, both effective diffusivity and viscosity were showed to strongly depend on the microstructure

\footnotetext{
^e-mail: pierre.rognon@sydney.edu.au

$\star \star$ e-mail: prashidha.kharel@sydney.edu.au

$\star \star \star$ e-mail: thomas.Miller@smec.com

$\star \star \star \star$ e-mail: itai.einav@sydney.edu.au
}

of the grains within the flow. One approach developed to model such dependencies consists in introducing a function of the solid fraction $\phi$ in each of the scalings (1) and (2). However, it was showed that these functions tend to diverge near a maximum packing fraction, which makes them difficult to measure accurately [2, 3]. Moreover, while the solid fraction is an elementary property of the flow microstructure, its signification in terms of internal kinematics and dynamics is limited. As a result, pinpointing the internal mechanisms underpinning both scaling, and thus both effective diffusivity and viscosity remains a great challenge.

An alternative approach developed to model and explain the dependencies of the viscosity and diffusivity in terms of flow microstructure consists in expressing these quantities as a function of a length scale $\ell$ as:

$$
\begin{aligned}
D & \propto \ell^{2} \dot{\gamma} \\
\nu & \propto \ell^{2} \dot{\gamma}
\end{aligned}
$$

where $\ell$ represents the spatial extent of some microstructural organisation of the grains. However, the precise nature of the microstructural process relevant to diffusivity and viscosity remains somewhat elusive.

In this paper we discuss the relevance of considering granular vortices as a microstructural entity to better understand the effective diffusivity and viscosity in dense granular flows. In section 2 , we present some observations and measurements of granular vortices in experimental and simulated granular flows. In section 3, we discuss 
how granular vortices may explain observations of shear rate independent diffusivities. In section 4, we discuss how granular vortices could help rationalising some observed non-local behaviour of the granular viscosity.

\section{Shear induced granular vortices}

Within dense granular materials subjected to shear, grain trajectory does not follow a laminar streamline. Instead, grains exhibit strong fluctuations of velocities [59]. These fluctuations were reported to form spatially correlated patterns in many different flow configurations. Here we focus on one particular quantity characterising these kinematic fields, the local vorticity $w$. This section presents experimental and numerical results with the aim of highlighting that, within dense granular flows, the local vorticity is highly heterogenous and present some degree of spatial correlation from which vortices can be identified.

\subsection{Stadium shear experiments}

The Stadium shear device, showed on Figure 1a, was designed to continuously shear an assembly of bidimensional grains in a plane shear geometry [10]. Grains are nylon cylinders $10 \mathrm{~mm}$ high, of diameters 12,15 , and $20 \mathrm{~mm}$. They are set vertically on a horizontal glass panel, and enclosed into a belt. The belt is set in motion by a motor/sprocket system, subjecting the central zone to a plane shear flow with a prescribe shear rate $\dot{\gamma}=V / H$; a system of constant force springs prescribes a normal stress $\sigma$, thereby prescribing the inertial number $I$ :

$$
I=\dot{\gamma} t_{i}=\dot{\gamma} d \sqrt{\frac{\rho}{\sigma}}
$$

where $\rho$ is the grain density. Grains can move under the sprocket and recirculate into the central region, enabling us to perform shear deformations as large as desired. Individual grain trajectories are measured during the flow using a particle tracking algorithm.

Figure 1a shows a snapshot of the field of velocity fluctuations of the grains during one experiment. In some locations, the velocity fluctuations seemingly form nearly close loops streamlines, delineating zones rotating coherently. Using a circulation-based method introduced in [11], it is possible to extract individual vortices from such velocity fields. Such vortices are represented on Figure 1a. These are characterised by a large distribution of sizes and short life time [11].

\subsection{DEM simulations}

Besides these experiments, a discrete element method was used simulate the plane shear flow between rough walls (see Figure 1b). Grains are subjected to a prescribed shear rate $\dot{\gamma}=V / H$ and normal stress $\sigma$. Grains are cohesionless discs that interact through elastic, dissipative and frictional contacts characterised by a Young's modulus $E=1000 \sigma$,
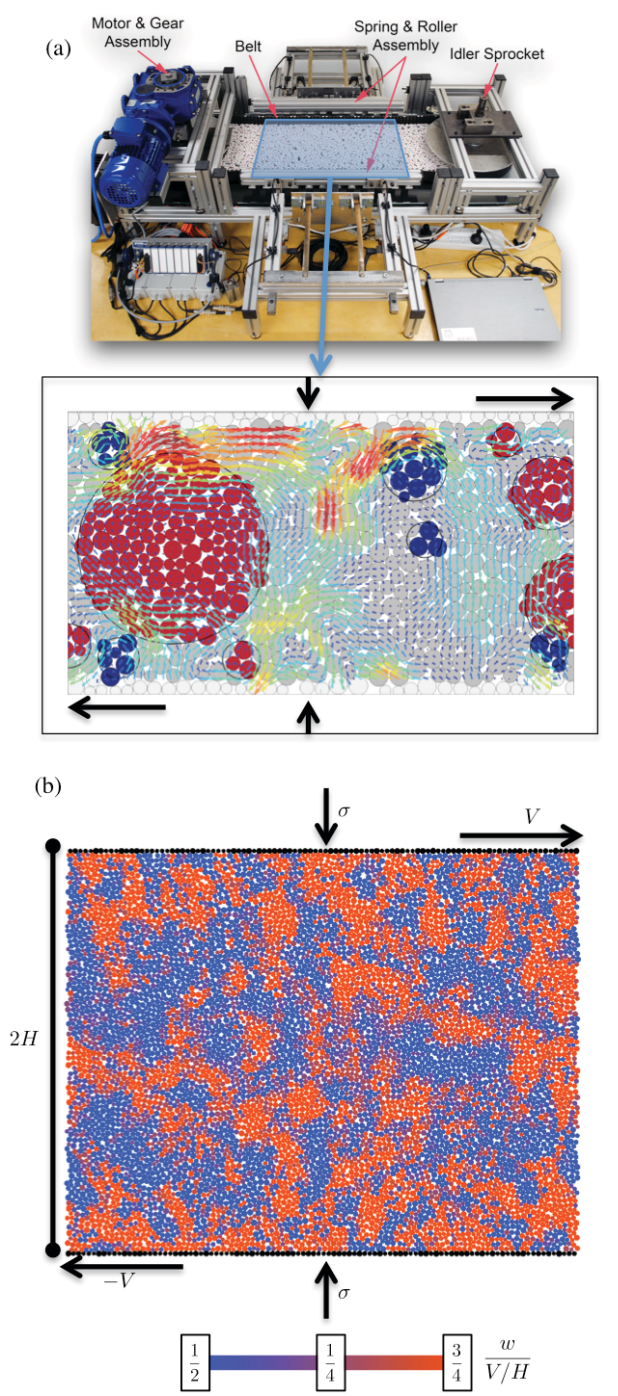

Figure 1. Granular vortices in plane shear of dense granular materials. (a) Granular vortices detected in flows produced by the Stadium Shear Device using a circulation-based method. Arrows denote individual grain velocity fluctuations, defined as the grain instantaneous velocity minus the time-average flow velocity at the grain location. Large blue circles denote detected vortices, including grains coloured in red for vortices rotating clockwise and blue for vortices rotating anti-clockwise. (b) Vorticity field measured in granular flow simulated with a discrete element method.

a coefficient of friction of 0.5 and a coefficient of normal restitution of 0.5 .

Figure $1 \mathrm{~b}$ shows a snapshot of the grains during the flow, coloured according to the level of vorticity in their surrounding. The local vorticity for each grains was measured as the skew-part of the local velocity gradient tensor, estimated using the neighbouring grains located within a distance of $2.5 d$ [12]. It appears that the local vorticity is not only highly heterogeneous, but also exhibits some spatial correlation, evidencing large patches of grains with a similar vorticity. This suggests that these patches of grains experience a nearly purely rotational rotation, with little or no shear. 


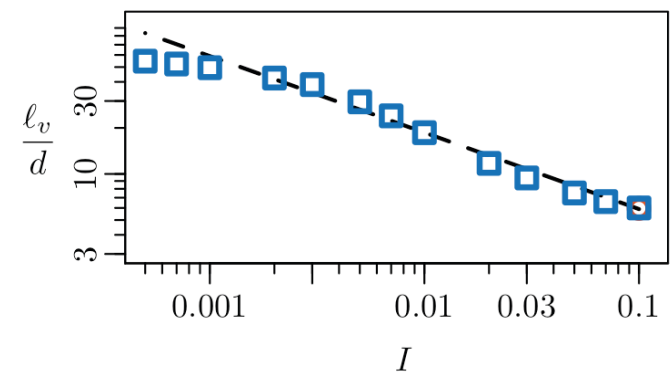

Figure 2. Average vortex size as a function of the inertial number, measured in simulated periodic plane shear flow $(H / d=$ 100). Symbols: simulation data; dashed line: $y \propto x^{-\frac{1}{2}}$

\subsection{Vortex size and inertial number}

A striking feature of granular vortices is that their average size within a flow depends on the inertial number. Figure 2 shows the average size of vortices measured in simulated plane shear flows at different inertial numbers. As a way to avoid wall effects, these simulation were performed using bi-periodic Lees-Edwards boundary conditions, as in [13]. On most of the explored range of inertial number, the average vortex size is found to scale like:

$$
\ell_{v} \propto \frac{d}{I^{\frac{1}{2}}}
$$

For too small inertial numbers, the size of the vortices seems to saturate, which we attribute to be an effect of the finite size of the simulated system: even in a periodic domain, vortices cannot be larger than $H$.

\section{Mixing}

An important consequence of the kinematic fluctuations in shear granular flows is that grains may move in the direction transverse to the flow. This shear-induced diffusion may be seen as resulting from a random walk, where grains move in the $y$ direction over a typical distance scaling like their own size $d$ at a frequency $\dot{\gamma}$. Such a random walk would lead to a effective diffusivity scaling like (2).

Figure 3 shows the grain diffusivity measured in a number of simulated flow in a periodic domain for different inertial numbers. While a linear dependency with $D$ and $\dot{\gamma}$ is evidenced at high inertial numbers, a breakdown of this scaling is observed at lower inertial number, with the diffusivity seemingly plateauing and becoming independent of the shear rate $[13,14]$. For even lower inertial number, the diffusivity becomes linearly dependent on the shear rate again.

These features may be explained by considering the vortex size as a typical length scale in (3). Accordingly, the diffusivity would scale like $D \propto \ell_{v}^{2} \dot{\gamma}$, or:

$$
\frac{D}{d^{2} / t_{i}} \propto\left(\frac{\ell_{v}}{d}\right)^{2} I
$$

Considering the scaling of the vortex size with the inertial number 6 would then lead to $D \propto d^{2} / t_{i}[15]$ and capture the shear rate independent behaviour of the diffusivity.
This scenario also predicts the recovery of a shear rate dependent viscosity at lower inertial number, given that the vortex size would then become limited by the system size and thus stop increasing as $I$ decreases.

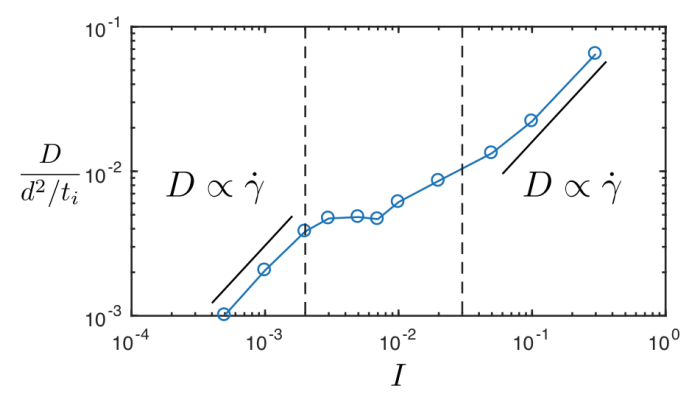

Figure 3. Effective diffusivity as a function of the inertial number measured in simulated plane shear flow in a periodic domain $(H / d=100)$. Symbols: data from DEM simulations; solid black lines: $y \propto x$; blue dashed lines: approximation of the regime where the diffusivity is nearly shear rate independent.

\section{Viscosity and non-locality}

The stadium shear experiments and the simulated plane shear flow between rigid walls presented in figure 1 produce homogeneous shear and normal stress across the sheared layer. Accordingly, assuming that the constitutive behaviour of the material is the same across the layer, one would expect that the shear rate $\dot{\gamma}(y)$ should be constant across the layer, $\dot{\gamma}(y)=V / H$. However, experimental and simulated flows exhibit a large variation of shear rate across the layer, as illustrated on figure 4 . The shear rate is much larger near the walls than in the central region. This indicates that the effective viscosity of the material is larger in the centre and smaller near the walls $[12,16]$.

Strikingly, the measured vortex size across the shear layer, $\ell_{v}(y)$, also exhibits large variations (see figure 4). Vortices are, in average, larger in the central region and smaller near walls. This can be attributed to a finite size effect: a vortex centred at a distance $Y$ from a wall cannot be larger than $Y$.

The correlation between vortex size variation and shear rate variation near walls suggests that vortices could be a microstructural entity affecting the viscosity of the material. In order to assess this possibility, we considered introducing the local vortex size as a typical length scale in the granular viscosity (4):

$$
v(y) \propto \ell_{v}^{2}(y) \dot{\gamma}(y)
$$

Applying the very definition of the viscosity, $v \propto \frac{\partial \tau}{\partial \dot{\gamma}}$ where $\tau$ is the shear stress, to this model leads to $\partial \tau \propto$ $\ell_{v}^{2}(y) \dot{\gamma}(y) \partial \dot{\gamma}$. Integrating along the transverse direction $y$ leads to the following relationship between the local shear rate and the vortex size:

$$
\frac{\dot{\gamma}(y)}{V / H} \propto \frac{d}{\ell_{v}(y)}
$$




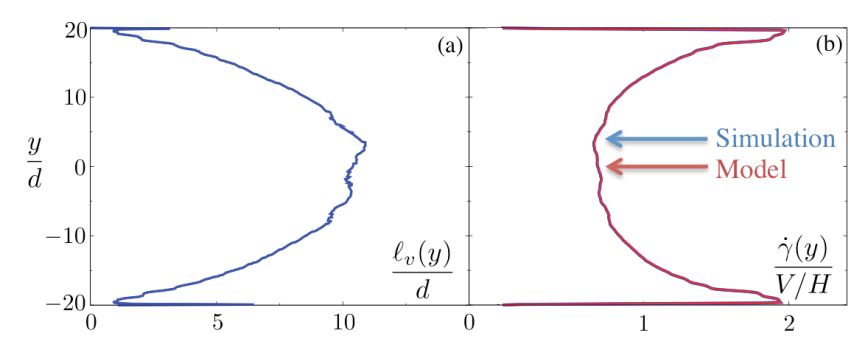

Figure 4. Granular vortices and effective viscosity. (a) Average granular vortex size measured at different location within a simulated plane shear flow between wall $\left(H=20 d, I=10^{-2}\right)$; (b) shear rate profile measured in the same flow, and predicted by using a viscosity based on the vortex length scale as per Eq. (8) (the two curves are on the top of each other) [12]).

Figure 4 shows that the shear rate profile predicted using (9) perfectly matches the numerical results. This supports the conclusion that vortices and their associated length scale may be an important microstructral parameter that influences the effective viscosity.

Beside long range wall effects, a number of non-local behaviours were observed in dense granular flows, as well as in dense flow of glassy materials, in presence of gradient of stress or shear rate. Different continuum non-local models were developed to capture such effects [12, 1720], which all rely on a characteristic length $\ell_{n l}$, sometimes referred to as cooperativity length, describing the spatial extent of the non-locality. Empirically, it was showed that this length should scale like:

$$
\ell_{n l} \propto \frac{d}{I^{\frac{1}{2}}}
$$

Strikingly, this scaling with the inertial number matches the measured scaling of the vortex size in (6). This suggests that vortices could potentially be a microstructural origin of non-local behaviour at the continuum scale. In a different paper part of these proceedings [21], we specifically focus on this connection and propose a mechanism that allows us to derive a continuum non-local fluidity model similar to that introduced in [19] simply by considering the existence of vortices.

\section{Conclusion}

The conclusion of this paper is that (i) there is a spontaneous development of spatially correlated vorticity within dense granular flows, and (ii) the resulting granular vortices may be a useful microstructural indicator to better understand and rationalise effective properties such as effective diffusivity and viscosity. Beyond granular materials, such analysis should be applicable to other glassy materials such as foams and emulsions, in which similar velocity fluctuations patterns have been observed.

While the purely kinematic analysis presented here has led to a number of useful insights, it is expected that it is not the only possible approach to explain the observed macroscopic behaviours. For instance, one could focus on other microstructural kinematic quantities such as granular temperature, or on dynamics properties such as force chains or distribution of local stresses. These approach could potentially lead to complementary insights and further the understanding of glassy material behaviour.

\section{References}

[1] Y. Forterre, O. Pouliquen, Annu. Rev. Fluid Mech. 40, 1 (2008)

[2] B. Andreotti, Y. Forterre, O. Pouliquen, Granular media: between fluid and solid (Cambridge University Press, 2013)

[3] C.S. Campbell, Journal of Fluid Mechanics 348, 85 (1997)

[4] J. Ottino, D. Khakhar, Annual Review of Fluid Mechanics 32, 55 (2000)

[5] F. Radjai, S. Roux, Physical review letters 89, 064302 (2002)

[6] O. Pouliquen, Physical Review Letters 93 (2004)

[7] M.R. Kuhn, K. Bagi, International journal of solids and structures 41, 5793 (2004)

[8] F. da Cruz, S. Emam, M. Prochnow, J.N. Roux, F. Chevoir, Physical Review E 72, 021309 (2005)

[9] G. Combe, V. Richefeu, M. Stasiak, A.P.F. Atman, Phys. Rev. Lett. 115, 238301 (2015)

[10] T. Miller, P. Rognon, B. Metzger, I. Einav, Physical Review Letters 111, 058002 (2013)

[11] P. Rognon, T. Miller, I. Einav, Granular Matter 17, 177 (2015)

[12] P.G. Rognon, T. Miller, B. Metzger, I. Einav, Journal of Fluid Mechanics 764, 171 (2015)

[13] P. Rognon, I. Einav, Physical Review Letters 105 (2010)

[14] Y. Fan, P.B. Umbanhowar, J.M. Ottino, R.M. Lueptow, Physical review letters 115, 088001 (2015)

[15] D. Griffani, P. Rognon, B. Metzger, I. Einav, Physics of Fluids (1994-present) 25, 093301 (2013)

[16] L. Staron, P.Y. Lagrée, C. Josserand, D. Lhuillier, Physics of Fluids (1994-present) 22, 113303 (2010)

[17] J. Goyon, A. Colin, G. Ovarlez, A. Ajdari, L. Bocquet, Nature 454, 84 (2008)

[18] O. Pouliquen, Y. Forterre, Philosophical Transactions of the Royal Society A: Mathematical, Physical and Engineering Sciences 367, 5091 (2009)

[19] K. Kamrin, G. Koval, Physical Review Letters 108, 178301 (2012)

[20] M. Bouzid, M. Trulsson, P. Claudin, E. Clément, B. Andreotti, Physical Review Letters 111, 238301 (2013)

[21] P. Kharel, P. Rognon, Partial jamming and nonlocality in dense granular flows, in Powders and Grains 2017 (2017) 